\title{
NGC 6441: another indication of very high helium content in globular cluster stars
}

\author{
V. Caloi ${ }^{1}$ and F. D'Antona ${ }^{2}$ \\ 1 INAF - Istituto di Astrofisica Spaziale e Fisica Cosmica-Roma, via Fosso del Cavaliere, 00133 Roma, Italy \\ e-mail: vittoria.caloi@iasf-roma.inaf.it \\ 2 INAF - Osservatorio Astronomico di Roma, via Frascati 33, 00040 Monte Porzio, Italy \\ e-mail: dantona@oa-roma.inaf.it
}

Received 20 July 2006 / Accepted 5 October 2006

\begin{abstract}
Context. The metal-rich bulge globular cluster NGC 6441 shows both a well-developed blue horizontal branch (Rich et al.), and a strong slope upward from the red clump to the blue of the RR Lyrae region. The former corresponds to the well-known second parameter problem. Both features are not explained by conventional evolutionary models.

Aims. Helium self-enrichment is proposed as a possible solution to both questions, a mechanism already invoked for the interpretation of the peculiarities in NGC 2808 and M 13.

Methods. We make use of horizontal branch simulations that cover a wide range of main sequence helium abundance to investigate whether the main features of the NGC 6441 horizontal branch population, including the RR Lyrae variables period, can be reproduced. Results. To describe the horizontal branch of NGC 6441, the following must be met. The helium content $Y$ in the red clump must reach at least 0.35 ; values up to $Y \sim 0.37$ are necessary to populate the RR Lyr region, also reproducing the observed mean long periods; depending on the dispersion in mass loss assumed in the simulations, values up to $Y \sim 0.38-0.40$ are necessary to populate the blue HB. The total self-enriched population amounts to $\sim 60 \%$ of the whole stellar content.

Conclusions. Self-enrichment and multiple star-formation episodes in the early evolution of globular clusters appear more and more able to account for many of the chemical and population peculiarities observed in these systems. The very large helium abundances ( $Y \gtrsim 0.35$ ) required for $\sim 14 \%$ of the horizontal branch population pose some problems for the enrichment mechanisms.
\end{abstract}

Key words. Galaxy: globular clusters: individual: NGC 6441 - stars: horizontal-branch - Galaxy: globular clusters: general

\section{Introduction}

In the past few years, some of the (by now) well known abundance anomalies in globular cluster (GC) star composition have been observed in unevolved cluster members (e.g., the anticorrelation between $\mathrm{Na}$ and $\mathrm{O}$ abundances; Gratton et al. 2001; Ramirez \& Cohen 2003; Cohen \& Melendez 2005; Carretta et al. 2006). These observations focused on the early stages of GC formation and evolution, since it appears that the abundance deviations from that of the pristine material took place before the formation of the stars in which they are observed.

A model that can explain all the chemical anomalies is not yet available, but some aspects of the problem begin to be understood, while others are still obscure. A clear step forward was made when the link was recognised between the chemical anomalies and the horizontal branch (HB) morphology of the clusters: if the chemical anomalies, and in particular the $\mathrm{Na}-\mathrm{O}$ anticorrelation, are interpreted in terms of a second generation of stars born from the ejecta of the massive asymptotic giant branch (AGB) stars of the first stellar generation, there must also be a helium discontinuity between the two samples of stars. In fact the AGB ejecta from which the second generation is born are all helium-enriched ${ }^{1}$ (D’Antona et al. 2002) and consequently the

1 The masses of the AGBs involved must be $\gtrsim 4 M_{\odot}$, otherwise the second generation would show evolutionary signatures of a prolonged phase of third dredge up; namely, the sum of $\mathrm{CNO}$ abundances would not be costant, and there would be s-process element enhancement. second generation stars evolving along the HB have bluer locations with respect to the first generation HB stars. This model was able to produce a detailed explanation of the anomalous morphology of the HB of the cluster NGC 2808 (D'Antona \& Caloi 2004); besides that, observations have confirmed the existence of a $\mathrm{Na}-\mathrm{O}$ anticorrelation in the cluster (Carretta et al. 2006).

An intriguing problem concerning only NGC 2808 and the "cluster" $\omega$ Cen at the moment is the presence of a well defined second main sequence (MS), bluer than the main one. In terms of standard MS models, this feature is a helium-rich sequence in which the helium mass fraction must be $Y \sim 0.4$ (see for $\omega$ Cen Norris 2004; Piotto et al. 2005; and for NGC 2808 D'Antona et al. 2005). The AGB models do not predict such a high helium abundance in the ejecta, the maximum computed value being $Y \simeq 0.36$ in Lattanzio et al. (2004), obtained from the evolution of a $6 M_{\odot}$ with $Z=0.004$. Ventura et al. (2002) quote values up to $Y \simeq 0.31$, and Ventura \& D'Antona (2005b) estimate $Y=0.32$ for their most massive models $\left(6.5 M_{\odot}\right)$ for metallicity $Z=0.001$. Although this value can be considered conservative, as these models do not include any kind of overshooting, a preliminary exploration of the second dredge up does not increase $Y$ beyond $\sim 0.35$ (Ventura 2006, private communication). The episodes of third dredge up may help to increase the helium content in some models (Ventura \& D'Antona 2005a), but

The helium content of the ejecta of a $4 M_{\odot}$ AGB star is $Y \sim 0.27$ for $Z=0.001$. 
too many episodes in the end do not preserve the constancy of $\mathrm{C}+\mathrm{N}+\mathrm{O}$. Inclusion of the results from these computations in a code for computing the chemical evolution of helium confirms that the present AGB models cannot explain the extreme helium enhancements (Karakas et al. 2006). The role of super-AGBs, the stars that ignite Carbon in semidegenerate conditions and may evolve through a AGB phase, ending up their lives as $\mathrm{O}_{-}$ $\mathrm{Ne}-\mathrm{Mg}$ white dwarfs, has not yet been explored for the metallicities of GCs. Siess (2006) finds $Y \sim 0.38$ for these stars after the second dredge up, but this result may be affected by the initial value of the helium content, as his models refer to solar metallicity.

The extreme helium enhancement, derived by D'Antona et al. (2005) both from the blue MS and from the extreme HB blue tails EBT2 and EBT3 (as defined in Bedin et al. 2000), concerns only $\sim 15 \%$ of the stars in NGC 2808 , and a similar fraction of stars is involved in the bluer MS in $\omega$ Cen.

We recall that the extreme value of the helium content, obtained from the MS fit, is rather uncertain. On the basis of our present understanding, extreme helium enhancements $(Y>$ 0.35) may derive either from the high-mass tail of AGBs (for which at present we do not yet have models, see above) or from the wind ejecta of the massive stars in the first stellar generation (Piotto et al. 2005; Prantzos \& Charbonnel 2006; Smith 2006). This suggestion would complicate the picture even more of star formation in the "simple" stellar population, as GCs were considered until a few years ago. It is then important to ascertain whether there are any other GC in which there is a clear indication of an extreme helium rich $(Y>0.35)$ component. In the absence of new information coming from other main sequences, we considered modelling the peculiar $\mathrm{HB}$ of the cluster NGC 6441, whose morphology and RR Lyr population (Rich et al. 1997; Layden et al 1999; Pritzl et al. 2000) are both a clear indication of the presence of helium enrichment, as already suggested by Sweigart \& Catelan (1998), D’Antona et al. (2002) and D'Antona \& Caloi (2004). The results obtained from this analysis can be extended to the cluster NGC 6388, which is very similar to NGC 6441 in metallicity, the peculiar HB morphology and RR Lyr periods.

We proceed as follows: Sect. 2 presents the observational data for NGC 6441 and the related problems. The theoretical background is given in Sect. 3, while Sect. 4 describes the comparison with the observations with a possible solution to the questions in Sect. 2. Some aspects of the given solution are examined in Sect. 5, and Sect. 6 gives the final discussion.

\section{The horizontal branch in NGC 6441}

NGC 6441 belongs to the metal-rich group of GCs $(Z \gtrsim 0.004)$. All these clusters have an HB confined to the red side of the RR Lyr region, with the exception of NGC 6441 itself and of its “twin cluster” NGC 6388 (Rich et al. 1997).

The CM diagram of NGC 6441 is shown in Fig. 1 (data from Piotto et al. 2002, dereddened by $E(B-V)=0.44)$. The red clump has a steep slope upward, which continues in the RR Lyrae and blue HB regions, with a difference of at least 0.6 mag between the top of the blue HB and the bottom of the red clump. The features to be explained are a) the presence of HB stars hotter than the red clump ones; b) the much larger tilt in luminosity, than expected from evolutionary models (f.e., Raimondo et al. 2002); c) the very long average period of the RR Lyr variables, which is unexpected based on the metal content ( $Z \geq 0.006$; Layden et al. 1999; Pritzl et al. 2001, 2003);

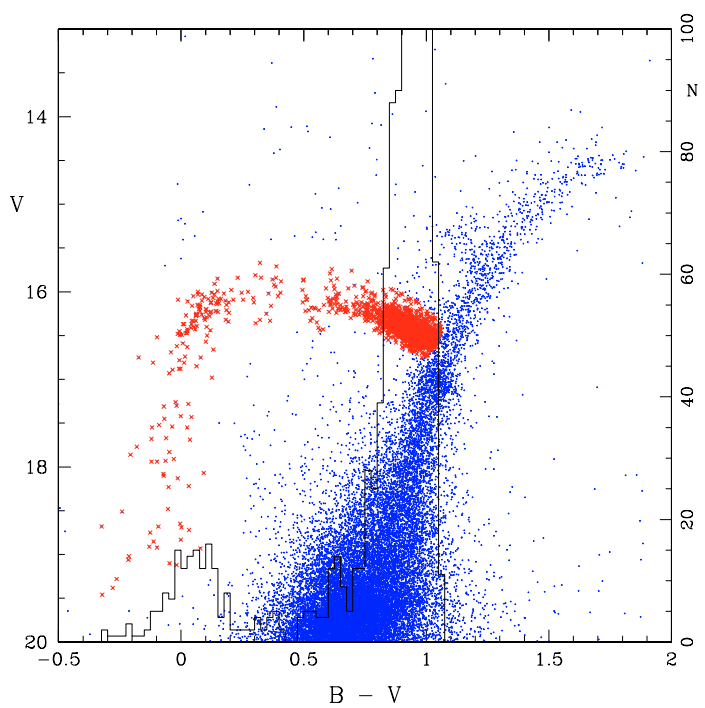

Fig. 1. CM diagram of NGC 6441 from the data by Piotto et al. (2002). The crosses (in red) indicate our selection of HB members; also shown is the histogram of their distribution with respect to $B-V$.

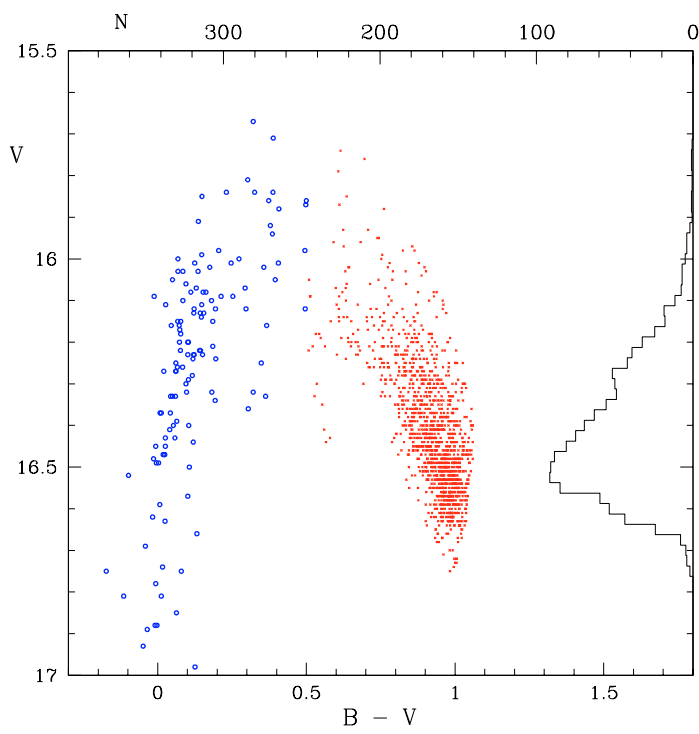

Fig. 2. An enlargement of the HB red clump region: crosses (red) indicate stars with $B-V>0.5 \mathrm{mag}$, open circles (blue) stars with $B-V<$ 0.5 mag. The histogram of red HB stars with respect to $V$ mag is shown.

d) the presence of an extended blue tail down to a magnitude $V \sim 19.5$, corresponding to $M_{v} \sim 3.5 \mathrm{mag}$.

In Fig. 1 our choice of the HB members is indicated. There are a total of 1452 members, out of which 1278 form a red clump $\left((B-V)_{0}>0.5\right)$. For our simulations we assumed therefore a total of 1452 stars, to be distributed according to observations. Also in Fig. 1 the histogram of the number of $\mathrm{HB}$ stars with respect to $B-V$ is shown; Fig. 2 shows the histogram with respect to $V$ mag, considering only stars with $(B-V)_{0}>0.5$.

There are 45 RR Lyr in the sample by Piotto et al. (2002) (Piotto, private communication). We assume a mean fundamentalized period (see below) of $0.67 \mathrm{~d}$, based on $63 \mathrm{RRab}$ and RRc by Pritzl et al. (2003). 


\section{The evolutionary and synthetic HB models}

The computation of HB models and of the synthetic HBs follows the prescription in D'Antona \& Caloi (2004). We model the HB by assuming cluster members to be a mixture of stars with different helium abundances, as we did for NGC 2808 and M 13. Previous simulations for the HB of NGC 6441 were performed by Sweigart \& Catelan (1998), who also considered models with high $Y$, but not a mixture of helium contents.

The scheme of our modelling is the following. We consider a first group of stars with a fixed helium abundance $Y$, close to the cosmological value $Y=0.25$, which represents the first stellar generation. A second (or more) generation(s) is added, with a helium content that varies from $Y \gtrsim 0.27$ to $Y \lesssim 0.40$. The number vs. helium distribution is chosen in order to comply with the relevant HB features mentioned in Sect. 2

\subsection{HB structures}

We computed evolutionary sequences from the MS to the red giant tip for a heavy element content of $Z=0.006$ and $Y=0.24$, $0.26,0.30$, and 0.40 . Having an estimate of the helium core masses at the flash, we obtained HB evolutionary sequences with the appropriate core masses and atmospheric helium abundances (after the first dredge-up). The theoretical tracks were transformed into Johnson magnitudes $B$ and $V$ by means of Bessell et al. (1998). The clump colours correspond nicely to the colours of the transformed tracks. Nevertheless, we do not emphasise this correspondence as a proof of a good choice of metallicity, as we could have shifted the observed colours within the uncertainties of the reddening ( \pm 0.03 according to Layden et al. 1999). Such an uncertainty has no influence on our comparisons, which rely quantitatively on luminosity differences and periods and only qualitatively on the colour extension of the $\mathrm{HB}$, which far exceeds the reddening error. HB simulations were computed in $V, B-V$, assuming a Gaussian error on magnitudes and colours. A standard deviation of 0.05 mag has been chosen, estimated from the HB data by Piotto et al. (2002).

\subsection{The main parameters in the simulations}

We assumed the ratio $\mathrm{R}$ of the number of blue $\left((B-V)_{0}<0.5\right)$ $\mathrm{HB}$ members to the total number of HB stars as the basic parameter describing the HB distribution. This ratio (174/1452 with the numbers above) is $R=0.12 \pm 0.01$. The other constraints to be satisfied by the simulations are the agreement with the histogram in Fig. 2, the extension in magnitude of the blue tail and the mean RR Lyr period.

In the case of the RR Lyr variables, we evaluated the mean period of the artificial RR Lyrae variables for every simulation by means of the van Albada \& Baker (1973) formulation; the limits of the RR Lyr region were taken from $\log T_{\text {eff }}=3.79$ to 3.875 . This is a standard interval for the metal-poorer GCs, and it may not correspond exactly to the present conditions of high $Z$ and high luminosity. The properties of the RR Lyraes in NGC 6441 appear extreme when compared with those of the whole family of GCs (see, e.g., Fig. 10 in Pritzl et al. 2001), but still within the limits of current interpretation. We are therefore confident that the order of magnitude of the estimated period is adequate. The observational mean period is built by averaging the observed periods, taking care to "fundamentalise" the periods of the RRc variables by adding 0.128 to the logarithm of their periods (van Albada \& Baker 1973).

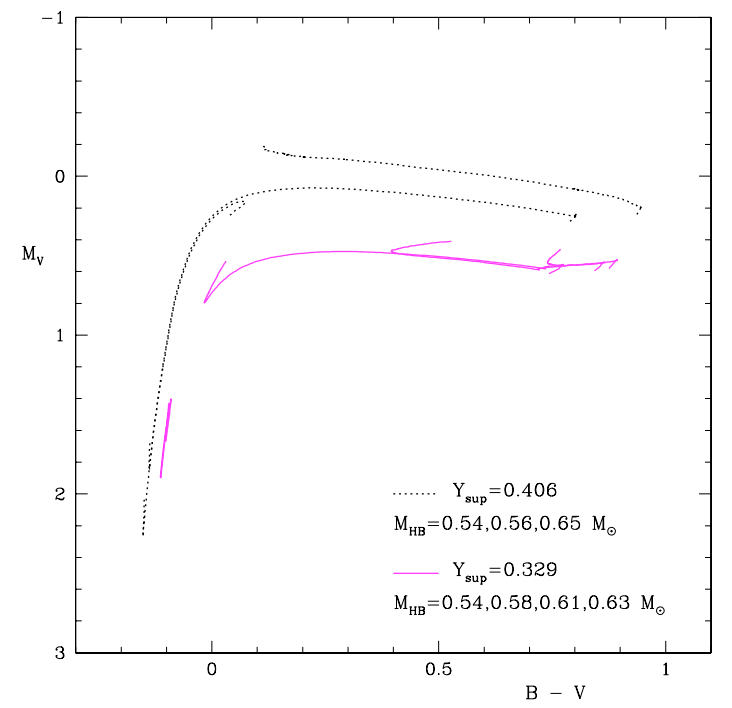

Fig. 3. Evolutionary tracks for a few helium-rich HB models: the long loops that they perform cover large portions of the allowed HB region. The tracks end at $Y_{\mathrm{c}}=0.10$.

\section{The simulations and the comparison with the data}

Two features, one observational and the other theoretical, strongly affect the comparison between observations and simulations:

1. the fact that the RR Lyrae variables and the blue component in NGC 6441 are only a tail of the red clump distribution, covering slighly more than $10 \%$ of the total HB population;

2. the behaviour in the HR diagram of $\mathrm{HB}$ models rich in helium.

The evolutionary path of the helium rich models may be confined in the red clump region, or may perform long loops, reaching very blue colours, for a difference in total mass of the order of $\lesssim 0.02 M_{\odot}$ : a well known fact, see, f.e., Sweigart \& Gross (1976). This is shown also in Fig. 3. Both these features conspire to vary noticeably the RR Lyr number and mean period, even by only varying the random extraction. Consequently, we first try to fit the observed fraction of stars at $B-V<0.5$ mag and impose the extension of the blue tail down to $M_{v}>3 \mathrm{mag}$. In any case, we do not accept a mean period smaller than $0.66 \mathrm{~d}$ for the RR Lyrs.

\subsection{Age, helium distribution and mass loss}

We assumed a reference age of 11 Gyr. Coeval populations with the same metallicity but different helium content will have turnoff masses that are inversely proportional to $Y$. Therefore, the progeny of the helium-rich, lower-mass evolving red giants will be located, on average, at higher temperatures (due to the lower evolving masses) and higher luminosity (due to the higher efficiency of the hydrogen-shell burning) than the structures with lower helium. To illustrate this basic fact, we show the behaviour of two groups of stars in Fig. 4. In one of these the maximum helium content is progressively increased. As expected, the evolutionary paths, confined within the red clump for lower $Y$ as happens for the cosmological helium population, reach farther and farther into the bluer HB regions when increasing the maximum $Y$.

For an age of $11 \mathrm{Gyr}$, we chose $\Delta M=0.165 M_{\odot}$ with $\sigma=0.02$ as mass loss during the red giant evolution. With this 

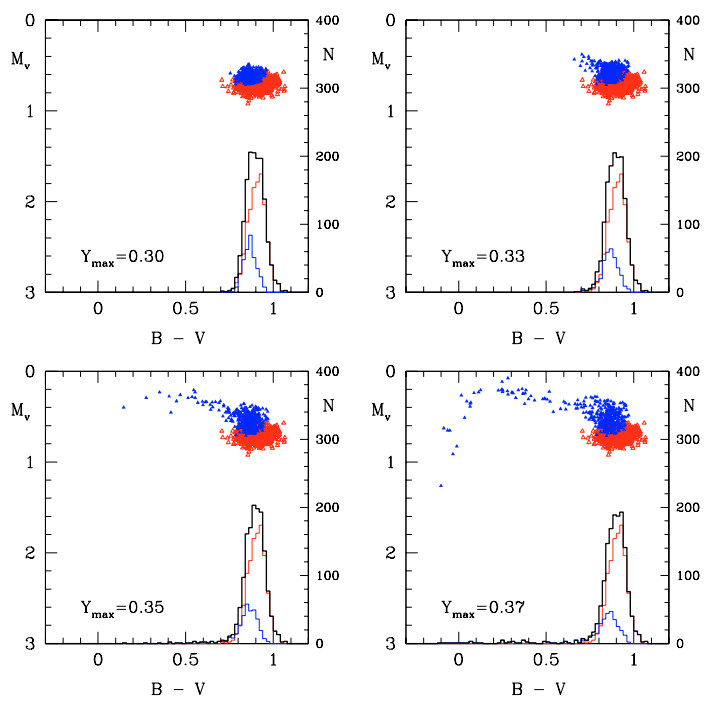

Fig. 4. Simulations show the effect on the evolutionary tracks of an increasing helium content: open triangles (red) indicate stars with fixed $Y=0.25$ (red histogram), filled triangles (blue) stars with increasing maximum helium content $\left(Y_{\max }\right)$ (blue histogram), as labeled in the four panels. The black histogram refers to the whole HB. $Z=0.006$ in all models.

amount it was easy to obtain the desired behaviour of the clump luminosity, when varying the helium content in the heliumenriched population(s). A larger mass loss would cause an excessive loss of clump members (increasing the fraction of stars at $B-V<0.5 \mathrm{mag}$ beyond the allowed values), while a lower one would have the opposite effect. Of course the value of $0.165 M_{\odot}$ is related to the chosen age: for $13 \mathrm{Gyr}$, a mass loss of $0.13 M_{\odot}$ would have given similar results.

The faintest blue HB stars in Fig. 1 - about 3.5 mag less luminous than the RR Lyrs - correspond to a HB mass $\leq 0.5 M_{\odot}$. For $Y=0.40$ and an age of $11 \mathrm{Gyr}$, the evolving red giant has a mass of $\sim 0.72 M_{\odot}$, which would require a mass loss $\geq 0.22 M_{\odot}$; larger mass losses would be necessary for lower $Y$, up to $0.45 M_{\odot}$ for $Y=0.25$. We therefore consider models with $Y$ up to 0.40 , but even at this large helium content the very faint magnitudes are statistically difficult to obtain. We took the possibility into account of an increase in the mass-loss rate with the decrease in the red giant mass, according to the Reimers (1977) mass-loss relation (see also Lee et al. 1994). But this is not an automatic solution to the problem; the progression in mass loss used in Lee et al. (1994) would not allow any HB progeny to red giants of 11 Gyr with the assumed $Z$, helium content 0.40 , and a basic mass loss of $0.165 M_{\odot}$ (for the case $Y=0.25$ ), so we assume a milder dependence of the mass loss on the evolving red giant mass. Another constraint is given by the necessity of keeping the required number of stars within the red clump (and with the observed distribution in luminosity), since, with increasing mass loss, the evolutionary tracks tend to shift to the blue (see Fig. 3).

In Figs. 5 and 6 we give an example of a synthetic HR diagram and of its detailed behaviour in the clump region. In addition to the parameters specified above - age $11 \mathrm{Gyr}, \Delta M=$ $0.165 M_{\odot}, \sigma=0.02$ - the extra mass loss included takes the form $0.10 \times\left[M_{\mathrm{rg}}(Y=0.25)-M_{\mathrm{rg}}(Y)\right]$. Here we discuss the main features of these diagrams.

To reproduce the strong upward tilt in the red clump, it is necessary to consider objects with a helium content up to $Y \sim$ 0.35. In Fig. 10 the typical relation of $B-V$ with $Y$ is shown. To get $B-V<0.5 \mathrm{mag}$, one needs stars with $Y>0.33$, while

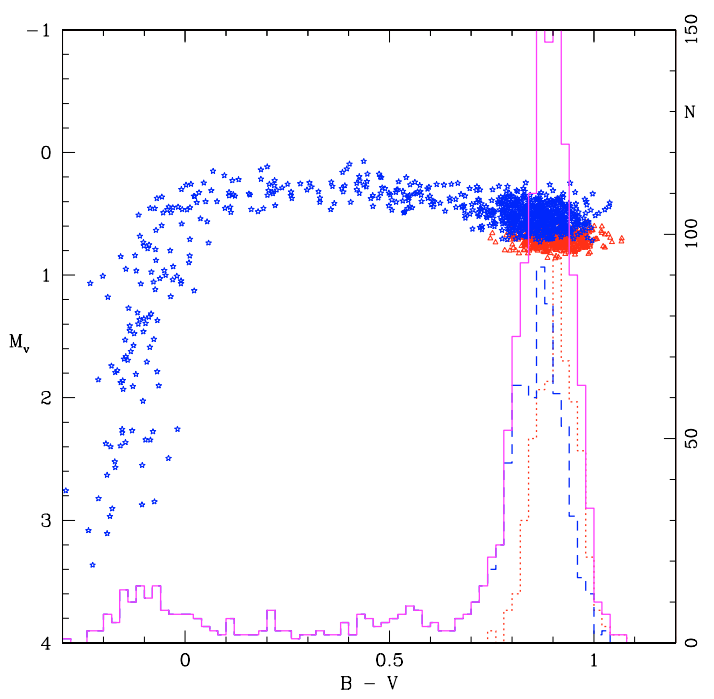

Fig. 5. A synthetic CM diagram of the HB in NGC 6441 for an age of $11 \mathrm{Gyr}$, mass loss of $0.165 M_{\odot}$ with $\sigma=0.02$, and extra mass loss as in the text. Stars with fixed $Y=0.25$ (open triangles, red) plus stars with variable $Y$ up to 0.40 (stars, blue). For the helium distribution, see text and the following figures. The standard deviation assumed for both $V$ and $B-V$ in this and in the following figures is $0.05 \mathrm{mag}$. The histograms give the number of stars as a function of $B-V$ : the total number is represented by the full line histogram, while the dotted one is the $Y=0.25$ population and the dashed one is the population with variable $Y$.

the main body of clump stars $(B-V>0.5)$ have a $Y$ content up to 0.37 ; we have to attain $Y>0.37$ to find (almost) exclusively blue stars.

In order to have, on the one hand, blue HB stars that are at least $\sim 3$ mag less luminous than the HB luminosity peak and, on the other, the expected number of red clump members, it is necessary to assume a non uniform $Y$ distribution for the second star generation. Otherwise, it is not possible to obtain a blue tail without remaining with too few red stars $(B-V>0.5)$.

The difference in helium content between the first and the second generations has to be $\sim 0.02$ (a gap in $Y$ from 0.25 to 0.27 ), so as not to increase the peak in the clump distribution (Fig. 7). At the same time, the gap cannot be larger than 0.03 ( $Y$ from 0.25 to 0.28 ), otherwise the clump distribution would show (always) two well-defined peaks (Fig. 8).

The importance of the assumed error on $V, B-V$ is exemplified in Fig. 9, where the same simulation of Fig. 6 is shown with an error on $V, B-V$ reduced from 0.05 to 0.03 mag: the clump appears clearly separated in two groups. This illustrates the relevance of improving the photometric precision in order to understand the population stratification in GCs better.

\subsection{Metallicity}

The adopted metallicity $Z=0.006$ falls on the lower side of the values acceptable for the cluster under exam (e.g. Clementini et al. 2005). In fact, it corresponds to the value on the Carretta $\&$ Gratton (1997) scale, if we do not consider $\alpha$-enhancement. On the other hand, this metal-rich cluster also seems to be $\alpha$-enhanced (Carretta, private communication). Should $Z$ be larger, all the estimated helium values would be pushed towards even higher values. 

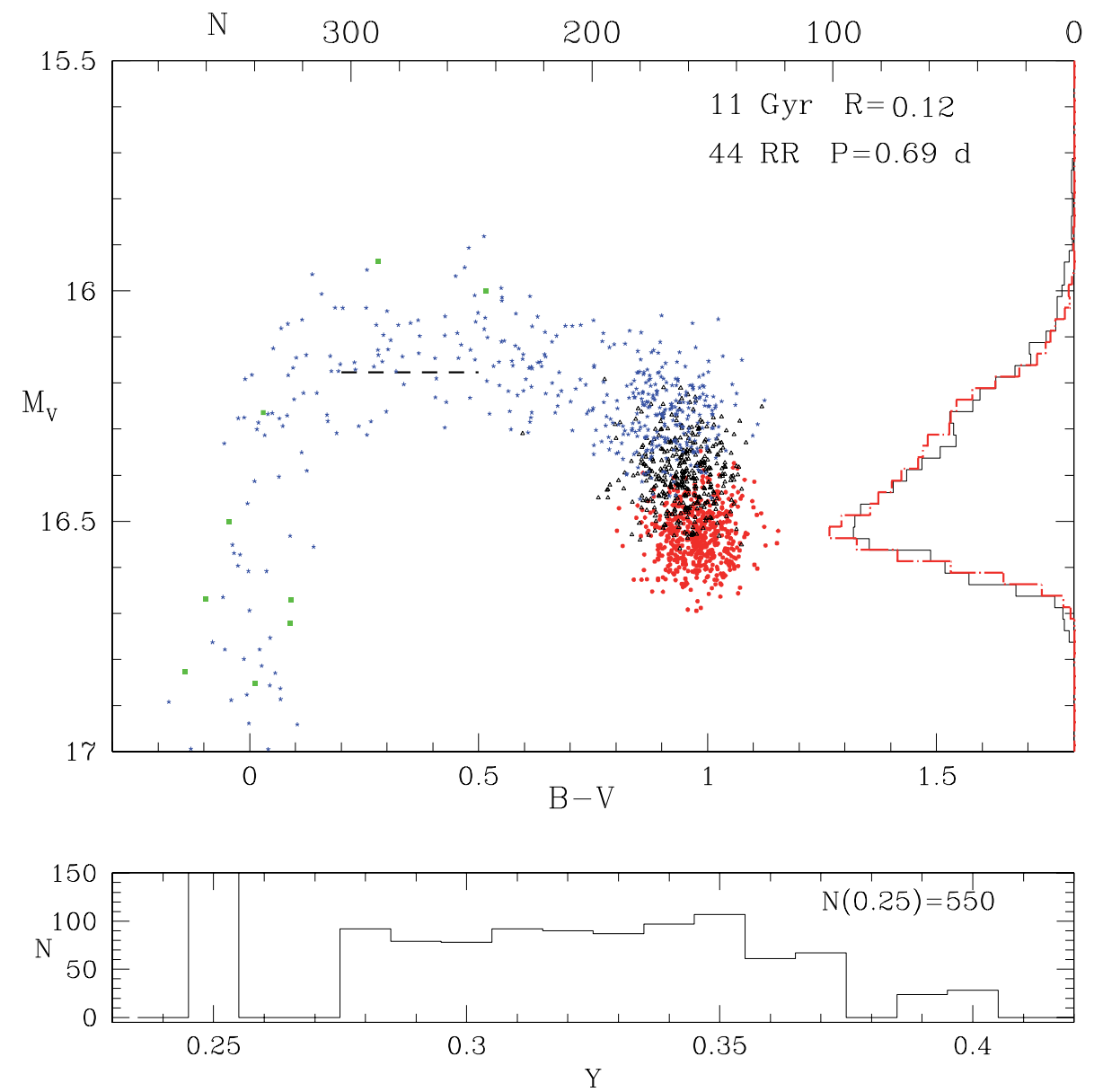

Fig. 6. For the simulation of Fig. 5, we show the synthetic CM of the red clump region in the top panel, with the details of the distribution in luminosity (line-dotted histogram, red). The continuous line gives the observed histogram, as in Fig. 2. Different symbols (and colours) denote different helium contents. Points: stars with $Y=0.25$ (red); open triangles (black): $0.27<Y<0.32$; asterisks (blue): $0.32<Y<0.38$; squares (green): $0.38<Y<0.40$. The dashed line indicates the approximate position of the RR Lyr variables, derived from Pritzl et al. (2003). The assumed age, the number of RR Lyr (RR), the average period of the RR Lyr (P), and the fraction of HB stars bluer than $B-V=0.5$ (R) in the simulation are listed at the top right. The lower panel shows the number distribution of HB stars vs. helium content corresponding to the CM diagram in the upper panel. The total number of the first stellar generation with $Y=0.25$ is given at the top right and labeled $\mathrm{N}(0.25)$.

\section{3. "The best solution"}

We consider to be satisfactory the result given by the simulation in Figs. 5 and 6. The helium distribution is shown in the lower panel of Fig. 6; an extra mass loss of $0.10 \times\left[M_{\mathrm{rg}}(Y=0.25)-\right.$ $\left.M_{\mathrm{rg}}(Y)\right]$ has been assumed, besides the standard $\Delta M=0.165 M_{\odot}$ mentioned before. The simulation has the correct value for the fraction of stars at $B-V<0.5$ mag within the errors, a number of RR Lyr variables close to the estimated one (45 in the sample in Piotto et al., as mentioned before) with a mean period of $0.69 \mathrm{~d}$ close to the observed fundamentalized period of $0.67 \mathrm{~d}$. Three very blue stars stars are found at $M_{v}>3.0 \mathrm{mag}$ in Fig. 5 .

We stress again that variables and BHB stars are only a tail of the cluster star distribution and that a small fluctuation in the clump number $\left( \pm \sqrt{(} N_{\text {clump }}\right)$ and in the number of stars that leave the clump at a slightly lower luminosity than the average, changes the number of RR Lyraes and their mean period noticeably.

\subsection{Dispersion in mass loss and maximum helium content}

In the simulations we adopt $\sigma=0.02$, a widely used value in the relevant literature (Lee et al. 1994; Catelan et al. 2001). With this assumption, it is necessary to include stars with $Y$ up to
0.40. It is important to see whether such extreme values of $Y$ can be avoided, since current models for possible self-enrichment in helium in globular clusters do not predict any value higher than $Y \simeq 0.36$. We performed simulations with higher values for $\sigma$ : while it is possible to achieve the end of the blue tail with $Y<0.4$, the mean RR Lyr period requires the presence of stars with $Y$ up to $\sim 0.37-0.38$. These stars in fact have the (large) luminosity necessary to obtain the long observed periods.

\subsection{The number vs. helium distribution}

The number of stars as a function of $Y$ assumed in the simulations is given in the bottom panels of Figs. 6-8. For our "best solution" we see that only 550/1452 38\% of stars belong to the first stellar generation with "normal" helium $Y=0.25$. Anoher important feature is made evident by Fig. 10. Stars with $Y>0.33$ are necessary to achieve $B-V<0.5 \mathrm{mag}$, but a large fraction of these objects remains in the red clump, due to the large excursions in colour of helium-rich structures (see Fig. 3). Besides, the presence of structures with $Y$ up to $\sim 0.37$ is required by the luminosity distribution of the clump itself (Fig. 6). This means that we must have a substantial amount of stars with $Y>0.33$ in the cluster. In fact, in our "best solution" (Sect. 4.3 

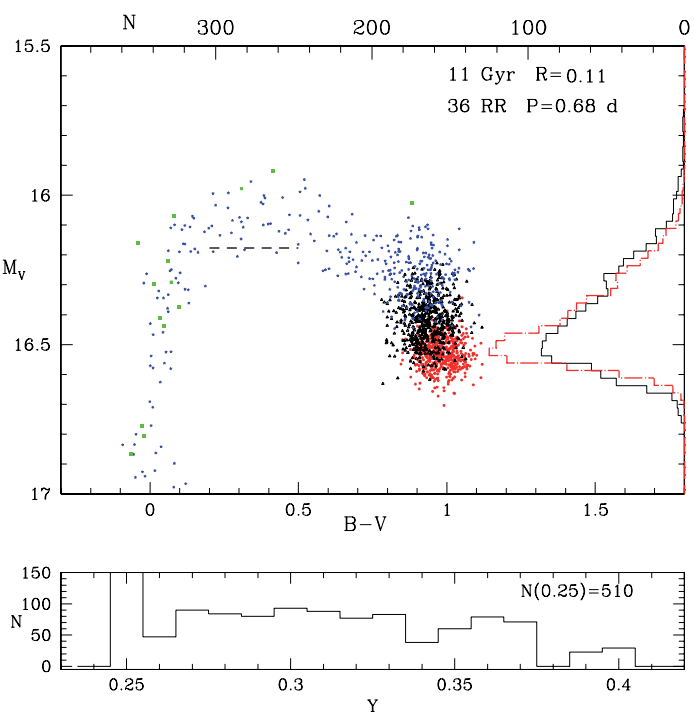

Fig. 7. As in Fig. 6, but with no gap in the $Y$ distribution (see the lower panel): it is not possible to avoid a substantially larger peak in the luminosity histogram than the one observed.


Fig. 8. As in Fig. 6, but with a gap of 0.035 between the $Y$ of the red clump and the lower limit of the population with varying $Y$ : two welldefined peaks appear in the luminosity histogram.

and Fig. 5), we have $\sim 38 \%$ of stars with $Y=0.25, \sim 33 \%$ of stars with $0.275 \leq Y \leq 0.33$, and $\sim 29 \%$ of stars with $Y>0.33$. About $15 \%$ of stars have $Y>0.35$. In this last group the objects are included with $Y=0.39-0.40(3.6 \%)$, which are the only ones to (almost) always remain at $B-V \leq 0.0$.

The exact numbers may be debated, as they depend in part on the age and mass loss along the RGB, and the extreme range $Y=0.39-0.40$ can be avoided with a mass dispersion $\geq 0.04 M_{\odot}$, but a substantial fraction $(\approx 30 \%)$ surely has $Y>0.33$.

\section{The $\boldsymbol{R}$ parameter and the bump location}

The ratio $R$ of the number of HB stars to the number of red giants above the HB level has been a powerful indicator of the helium content in cluster stars, as first suggested by Iben (1968a), when the aim was to distinguish the helium mass fraction in the oldest
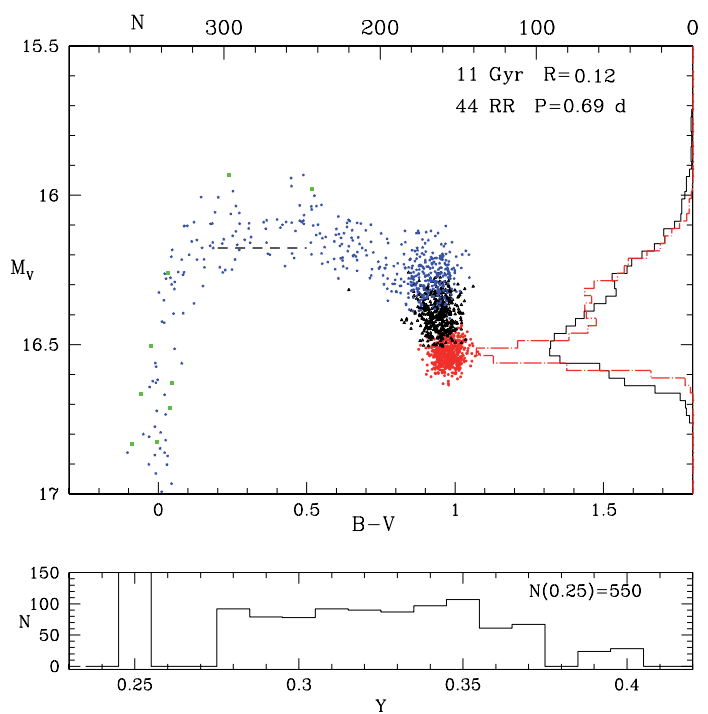

Fig. 9. As in Fig. 6, except that the standard deviation on $V$ and $B-V$ is reduced from 0.05 to 0.03 mag: the clump appears separated in two groups.

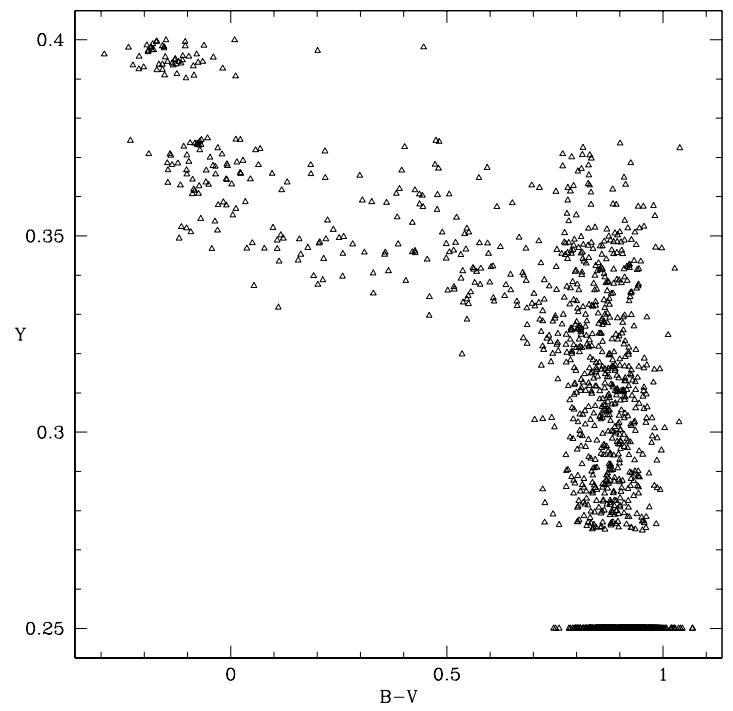

Fig. 10. The relation between $B-V$ and helium content $Y$ for the case in Fig. 6, but a quite similar behaviour has been found in all the simulations.

stars of the Galaxy between values close to $Y=0.1$ or to $Y=0.3$. For NGC 6441, Salaris et al. (2004) find a value $R=1.85 \pm 0.11$, which is consistent with the theoretical expectation by the same authors for the cluster metallicity. In contrast, the simulations by Sweigart \& Catelan (1998) predicted values as high as 3.43.9, when they assumed MS helium abundances of $Y=0.38$ and $Y=0.43$ for all the cluster stars. For these values, the level of luminosity of the HB clump is highly enhanced and thus the number of red giants to be considered is reduced. This in fact is also predicted by the simple interpolation formula by Buzzoni et al. (1983)

$Y=0.380 \log R+0.176$.

We do not explicitly compute the value of $R$ from our simulations, because it is easy to understand that we would find a "normal" value for $R$, due to the fact that the HB level in the $R$ definition has the zero age value, which in this cluster is determined by 
the stars having the normally adopted value of $Y=0.25$. These are the lowest luminosity stars in the red clump, and so the number of red giants to be considered remains the same as in the case with constant $Y=0.25$. In addition, there is only a small percentage of very hot stars with very high helium, whose HB lifetimes would exceed the $Y=0.25$ lifetimes, so that the correction to $R$ due to these stars would turn out to be small. Therefore, the argument by Layden et al. (1999) based on the $R$ parameter cannot be considered a proof that a high helium content does not explain the RR Lyr long periods.

Raimondo et al. (2002) noticed that a uniform high helium abundance of the cluster stars predicts the wrong location for the RGB bump (Thomas 1967; Iben 1968b) with respect to the HB. Also in this case, our modeling is consistent with this latter parameter: a prominent RGB bump will occur at the luminosity predicted by models with $Y=0.25$, which is the abundance of $\sim 40 \%$ of the stars. The stars with larger $Y$ will produce smaller bumps along the RGB at increasing luminosities, according to the number vs. $Y$ distribution. Notice also that the prominence of the bump decreases with increasing $Y$. Further, the HB reference luminosity level in our simulations, as mentioned above, corresponds to the $Y=0.25$ level. Therefore, the quoted difficulty with a high-helium population by Raimondo et al. (2002) does not apply to our model.

\section{Discussion}

Analysing the HB morphology of NGC 6441 in terms of a helium-rich population has provided a coherent interpretation of the main features of the anomalous HB of this cluster:

1. the long extension in luminosity of the red clump;

2. the fact that RR Lyrs have a very long average period, which is unusual for a cluster of high metallicity;

3 . the extension into the blue of the HB.

Modellization shows that a continuous distribution of helium content above $Y=0.25$ is not consistent with the luminosity distribution of stars in the red clump and also that a helium discontinuity must be present from $Y=0.25$ to a minimum value of $Y \simeq 0.27$, as in the cluster NGC 2808. We suggest that more precise photometry of the $H B$ would prove a double peak in the luminosity distribution of the red clump stars.

While it has been long suspected that the blue HB and the high RR Lyr luminosity in this cluster - and in its twin NGC 6388 - could be easily attributed to the high helium content in a small fraction of stars - 12\% - constituting the blue HB (Sweigart \& Catelan 1998; D’Antona \& Caloi 2004), one surprise from these detailed simulations is that a much larger fraction of stars has a very large $Y: 29 \%$ have $Y>0.33$ and $\sim 14 \%$ have $Y>0.35$. This latter figure is similar to the percentage of stars with $Y \sim 0.4$ found from analysing the MS in NGC 2808 (D'Antona et al. 2005). In this respect, we conclude that both NGC 6441 and NGC 2808 were able to form about the same fraction of very high helium stars, whatever their origin.

The difference between the two clusters resides in the intermediate helium population, which for NGC 2808 contains $35 \%$ of stars, and covers only the range between 0.26 and 0.29 (D'Antona et al. 2005). In NGC 6441, this fraction is $\sim 48 \%$, and it covers a helium content from 0.27 to 0.35 . Thus, any dynamical and chemical evolution model for these clusters must also explain the difference in this intermediate population. We suggest that it is due to the different metallicities of the two clusters: in NGC $2808\left(Z \sim 1-2 \times 10^{-3}\right)$ the maximum mass for AGB type evolution is lower than in NGC $6441\left(Z \gtrsim 6 \times 10^{-3}\right)$. Therefore, the range of progenitor masses for the second stellar generation in NGC 6441 contains stars of higher mass that also have higher helium content (Ventura et al. 2002).

One of the motivations for pursuing the present work was to investigate whether an "extreme" high-helium population is present in other clusters besides NGC 2808 and $\omega$ Cen. We found this extreme helium population in NGC 6441, which must alsoexist in the very similar cluster NGC 6388, and we also discovered that more than $60 \%$ of the cluster stars belong to the second stellar generation, with $0.27 \lesssim Y \lesssim 0.4$. This interpretation of the HB morphology of NGC 6441 reinforces the importance of understanding which is the origin of these extreme helium-rich stars.

Acknowledgements. We thank Dr. Anna Marenzi for help preparing this paper and Prof. G. Piotto for providing the database for NGC 6441.

\section{References}

Bedin, L. R., Piotto, G., Zoccali, M., et al. 2000, A\&A, 363, 159

Bessell, M. S., Castelli, F., \& Plez, B. 1998, A\&A, 333, 231

Buzzoni, A., Pecci, F. F., Buonanno, R., \& Corsi, C. E. 1983, A\&A, 128, 94

Carretta, E., \& Gratton, R. G. 1997, A\&AS, 121, 95

Carretta, E., Bragaglia, A., Gratton, R. G., et al. 2006, A\&A, 450, 523

Catelan, M., Ferraro, F. R., \& Rood, R. T. 2001, ApJ, 560, 970

Clementini, G., Gratton, R. G., Bragaglia, A., et al. 2005, ApJ, 630, L145

Cohen, J. G., \& Meléndez, J. 2005, AJ, 129, 303

D'Antona, F., \& Caloi, V. 2004, ApJ, 611, 871

D’Antona, F., Caloi, V., Montalbán, J., Ventura, P., \& Gratton, R. 2002, A\&A, 395, 69

D’Antona, F., Bellazzini, M., Caloi, V., et al. 2005, ApJ, 631, 868

Gratton, R. G., Bonifacio, P., Bragaglia, A., et al. 2001, A\&A, 369, 87

Iben, I., Jr. 1968a, Nature, 220, 143

Iben, I., Jr. 1968b, ApJ, 154, 581

Karakas, A., Fenner, Y., Sills, A., Campbell, S., \& Lattanzio, J. 2006, [arXiv:astro-ph/0605540]

Lattanzio, J., Karakas, A., Campbell, S., Elliott, L., \& Chieffi, A. 2004, Memorie della Societa Astronomica Italiana, 75, 322

Layden, A. C., Ritter, L. A., Welch, D. L., \& Webb, T. M. A. 1999, AJ, 117, 1313

Lee, Y., Demarque, P., \& Zinn, R. 1994, ApJ, 423, 248

Norris, J. E. 2004, ApJ, 612, L25

Piotto, G., King, I. R., Djorgovski, S. G., et al. 2002, A\&A, 391, 945

Piotto, G., Villanova, S., Bedin, L. R., et al. 2005, ApJ, 621, 777

Prantzos, N., \& Charbonnel, C. 2006 [arXiv: astro-ph/0606112]

Pritzl, B., Smith, H. A., Catelan, M., \& Sweigart, A. V. 2000, ApJ, 530, L41

Pritzl, B. J., Smith, H. A., Catelan, M., \& Sweigart, A. V. 2001, AJ, 122, 2600

Pritzl, B. J., Smith, H. A., Stetson, P. B., et al. 2003, AJ, 126, 1381

Raimondo, G., Castellani, V., Cassisi, S., Brocato, E., \& Piotto, G. 2002, ApJ, 569,975

Ramírez, S. V., \& Cohen, J. G. 2003, AJ, 125, 224

Reimers, D. 1977, A\&A, 61, 217

Rich, R. M., Sosin, C., Djorgovski, S. G., et al. 1997, ApJ, 484, L25

Salaris, M., Riello, M., Cassisi, S., \& Piotto, G. 2004, A\&A, 420, 911

Siess, L. 2006, A\&A, 448, 717

Smith, G. H. 2006, PASP, 118, 1225

Sweigart, A. V., \& Gross, P. G. 1976, ApJS, 32, 367

Sweigart, A. V., \& Catelan, M. 1998, ApJ, 501, L63

Thomas, H.-C. 1967, Z. Astrophys., 67, 420

van Albada, T. S., \& Baker, N. 1973, ApJ, 185, 477

Ventura, P., \& D'Antona, F. 2005a, A\&A, 431, 279

Ventura, P., \& D'Antona, F. 2005b, ApJ, 635, L149

Ventura, P., D'Antona, F., Mazzitelli, I., \& Gratton, R. 2001, ApJ, 550, L65

Ventura, P., D’Antona, F., \& Mazzitelli, I. 2002, A\&A, 393, 215 